\title{
Status of the OLYMPUS Experiment at DESY
}

\author{
M. Kohl for the OLYMPUS collaboration \\ Hampton University, Hampton, VA 23668 and Jefferson Lab, Newport News, VA 23606, USA
}

\begin{abstract}
The OLYMPUS experiment at DESY, Hamburg, Germany will definitively determine the effect of two-photon exchange in elastic lepton-proton scattering by precisely measuring the ratio of positron-proton to electron-proton elastic unpolarized cross sections. In this paper, the physics motivation is summarized, aspects of the experimental setup are discussed and the current status and timeline of the project are reviewed.
\end{abstract}

Keywords: Form factor, two photon exchange, positron, electron

PACS: 13.40.-f, 13.40.Gp, 13.88.+e, 14.20.Dh, 25.30.Bf

Recent determinations of the proton electric to magnetic elastic form factor ratio from polarization transfer measurements at Jefferson Lab [1,2] have indicated an unexpected and dramatic discrepancy with the elastic form factor ratio obtained using the Rosenbluth separation technique in unpolarized cross section measurements [3]. The discrepancy has been explained as the effect of hard two-photon exchange beyond the usual one-photon exchange approximation in the calculation of the elastic electron-proton scattering cross section. [5, 6, 7, 8, 9, 10,11]. The results from both methods are mostly based on the single photon exchange assumption including standard radiative corrections [4], which account for two-photon exchange only to the extent that one of the photons is soft.

Most of our understanding of the structure of the nucleon and of nuclei is based upon lepton scattering analyzed in terms of the single photon approximation, hence it is essential to precisely quantify the effect from the exchange of two and more photons. Contrary to standard radiative corrections of the lepton arm, which are calculable based on pure QED principles, calculations of the two-photon exchange amplitude are necessarily model-dependent in order to describe the intermediate off-shell hadronic state between the two photon vertices. Such calculations have been carried out e.g. in hadronic [7, 8], or partonic frameworks of generalized parton distributions and perturbative QCD $[9,10]$. Also the importance of higher-order radiative effects, not necessarily through two or more photons, has been emphasized [12].

The general structure of two-photon exchange introduces three new complex amplitudes [11]. While the imaginary parts give rise to small single-spin asymmetries, which can be measured with transversely polarized electron beam, transversely polarized target, or induced transverse recoil polarization, the real part of the two-photon exchange amplitude can manifest itself indirectly in a modified, linear or non-linear dependence on the virtual photon polarization $\varepsilon$ of the elastic cross section or polarization transfer. In particular, the real part of the two-photon amplitude matters for proton form factor extractions. While most calculations tend to remove the discrepancy in the form factor data, different model assumptions generally do not lead to a consistent determination of the individual amplitudes. For a satisfactory solution for the concurrent interpretation issue of lepton scattering experiments, it is essential to definitively verify the contribution of multiple photon exchange.

The only stringent observable that directly probes the size of the two-photon exchange amplitude is the difference of unpolarized elastic electron-proton and positron-proton cross sections. Experimentally, it is more advantageous to measure the ratio of such cross sections. To leading order, the interference of the single and two-photon amplitudes yield a contribution $\propto \alpha^{3}(\alpha=1 / 137)$ to the cross section. This interference effect is odd in the number of lepton vertices and therefore changes its sign when switching between electron and positron probes. In the presence of twophoton exchange, the $e^{+} / e^{-}$cross section ratio will deviate from unity. This effect is expected to be angular dependent, to increase with the scattering angle, likewise to decrease with $\varepsilon$, and to increase with $Q^{2}$, in order to explain the form factor discrepancy. The effect disappears for $\varepsilon \rightarrow 1$. Existing measurements of the ratio were carried out in the 1960's without showing significant evidence for an effect [13], as displayed in Fig. 2. Most of these data were however measured either at low $Q^{2}$ or at large $\varepsilon$, where the effect is also expected to be small.

The OLYMPUS experiment has been proposed at the storage DORIS at DESY [14]. It aims to precisely measure the ratio of elastic $e^{+} p$ and $e^{-} p$ scattering cross sections to better than $1 \%$ total error in a kinematic region where the ratio 
is expected to deviate from 1 on the order $5-10 \%$, if two-photon exchange is indeed responsible for the form factor discrepancy between Rosenbluth and recoil polarization measurements. OLYMPUS has been designed to minimize systematic and statistical uncertainties

- by running the experiment at the DORIS storage ring at DESY, Hamburg, Germany, which provides both electron and positron beams with rapid switching between both running modes, each with currents of $>100 \mathrm{~mA}$,

- by scattering leptons from an internal, unpolarized, isotopically pure, essentially background-free hydrogen gas target of $3 \cdot 10^{15}$ atoms $/ \mathrm{s}$, resulting in a luminosity of $2 \cdot 10^{33} /\left(\mathrm{cm}^{2} \mathrm{~s}\right)$,

- by deploying the available symmetric, large acceptance detector BLAST from the MIT-Bates Linear Accelerator Center [15, 16, 17], which features a toroidal magnetic field allowing to rapidly switch between both polarities and excellent tracking capability over a wide range of scattering angles of $\approx 20^{\circ}-80^{\circ}$ and $\pm 15^{\circ}$ out of plane,

- and by redundantly measuring the relative luminosity of OLYMPUS between electron and positron running and alternating toroidal magnet polarity based on small-angle elastic ep and symmetric Moller/Bhabha scattering.

OLYMPUS has been proposed at DESY in summer 2008 and was conditionally approved by the DESY Physics Research Committee (PRC) in October 2008. After a technical review in September 2009, main funding from the US agencies DOE and NSF has been secured in fall 2009, followed by the official approval of OLYMPUS by DESY in January 2010. Preparations of the experiment were started immediately, with the cleanup of the experimental area at DORIS and the transfer of the BLAST detector components to DESY completed in summer 2010. The detector has since been reassembled and will be finally mounted into the DORIS ring by mid 2011. Assembly and commissioning in park position can be carried out simultaneously with regular synchrotron operations of DORIS. The internal target has been newly designed and constructed at MIT using a customized target cell from INFN Ferrara. The new target will be installed early in 2011 and commissioned during DORIS synchrotron operations. Luminosity monitoring systems are under construction at Hampton University (GEM detector telescopes), Petersburg Nuclear Physics Institute (multiwire proportional chambers, MWPC), and at Mainz University (symmetric Moller/Bhabha counter arrays). A total of three months of production running at a beam energy of $2.0 \mathrm{GeV}$ and a luminosity of $L=2 \cdot 10^{33} /\left(\mathrm{cm}^{2} \mathrm{~s}\right)(500 \mathrm{~h}$ at $100 \mathrm{~mA}$ in each beam species) is required to provide the statistical accuracy of $<1 \%$ up to $Q^{2} \leq 2.2(\mathrm{GeV} / \mathrm{c})^{2}$. It is noted that the region near $Q^{2}=2.5(\mathrm{GeV} / \mathrm{c})^{2}$ is of particular interest, where the two-photon amplitude has recently been experimentally overdetermined $[2,6]$. Commissioning of the experiment at the standard operating energy of 4.5 $\mathrm{GeV}$ will provide an additional precise measurement of the cross section ratio at a large value of $\varepsilon \approx 0.9$ at a similar value of $Q^{2}$ in a fraction of the time, which will allow to infer a Rosenbluth-like separation of the $e^{+} p$ cross section by assuming the known $e^{-} p$ cross section. In the present scenario, production running of OLYMPUS will be completed

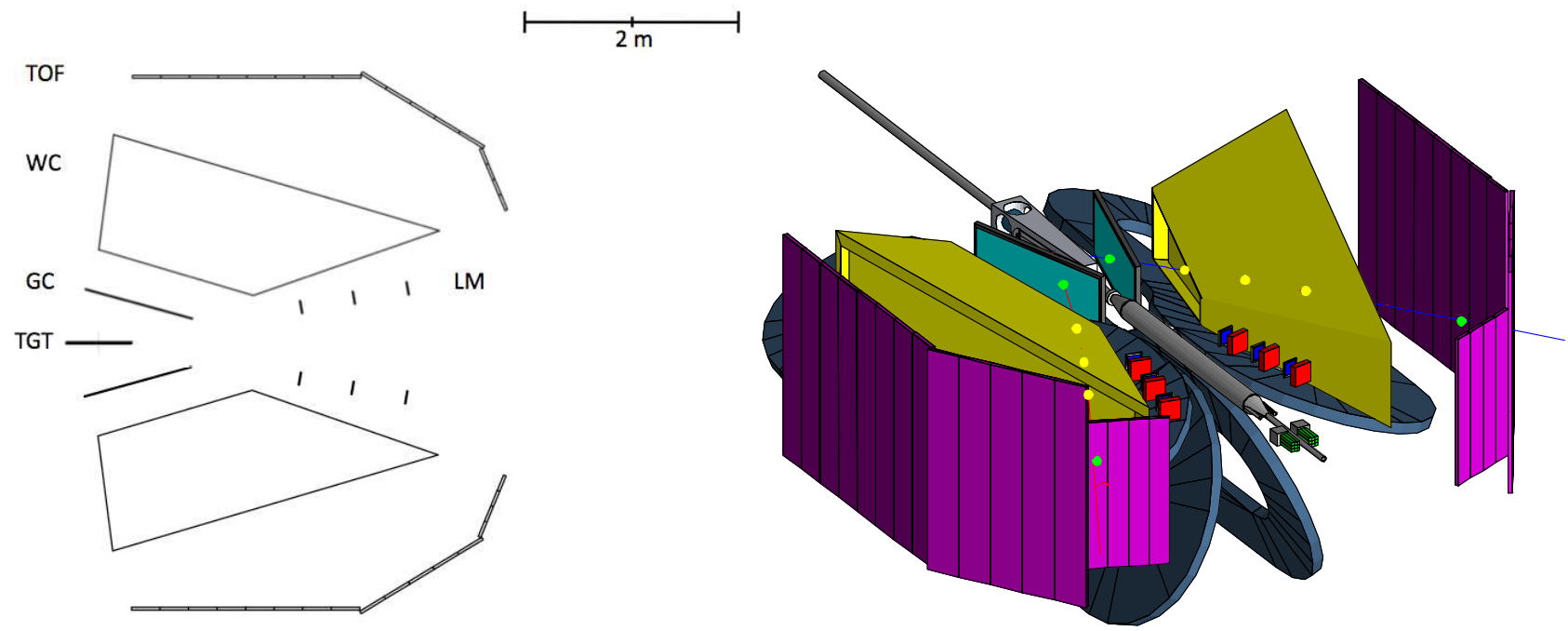

FIGURE 1. Left: Schematic layout of OLYMPUS in top view, with the beam coming from the left passing through the extended internal target. The displayed components are Time-of-Flight Scintillators (TOF), Wire Chambers (WC), Gas Electron Multiplier Chambers (GC), Luminosity Monitors (LM) and Target (TGT). In addition, there will be Moller/Bhabha monitors near the beamline for redundancy in luminosity monitoring. Right: Three-dimensional view of the OLYMPUS detector in Geant4. 


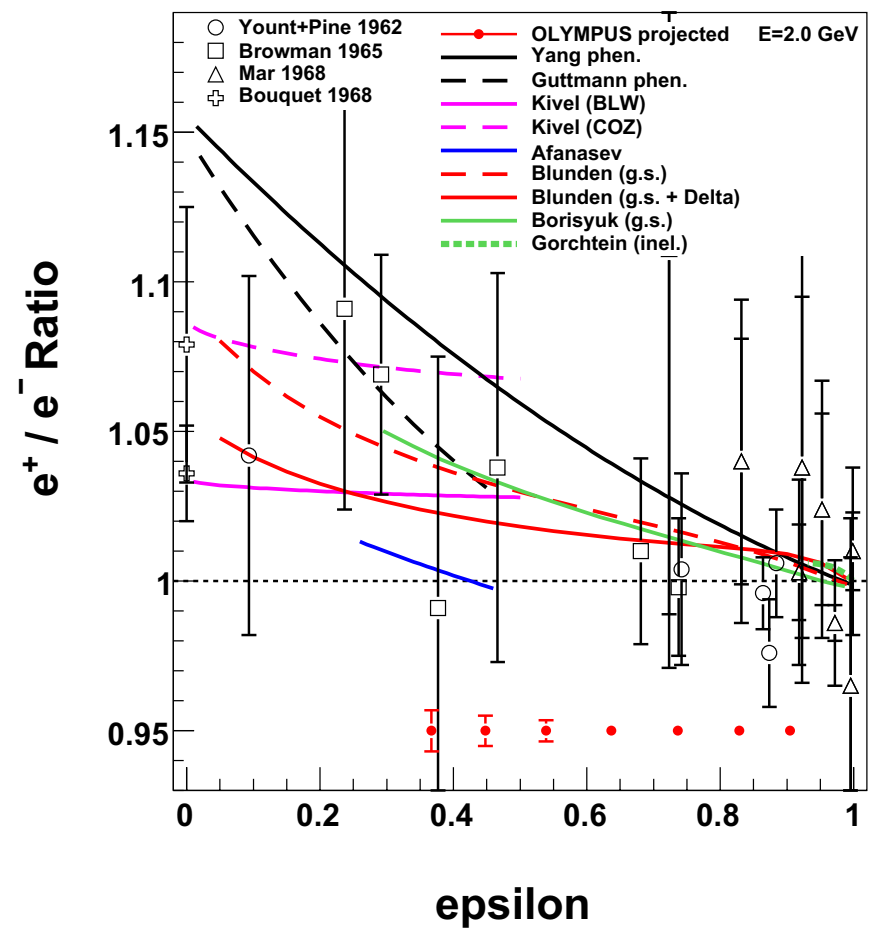

FIGURE 2. Projected precision of OLYMPUS for the $e^{+} p$ to $e^{-} p$ cross section ratio versus $\varepsilon$ along with previous data [13] and various theoretical predictions $[5,6,7,8,9,10,11]$, which have been evaluated for a constant beam energy of $\mathrm{E}=2 \mathrm{GeV}$.

by end of 2012. It should be noted that besides OLYMPUS, there are two proposals for experiments to measure the same cross section ratio, yet at slightly different kinematics (generally lower in $Q^{2}$ ) with different techniques and systematics, at the VEPP-3 storage ring in Novosibirsk [18] (data taking started in fall 2009) and at Jefferson Lab [19] (scheduled to run in 2011).

The schematic layout of OLYMPUS is shown in Figure 1. Of the original BLAST setup [15], the toroidal magnet, the wire chambers (WC) and the time-of-flight scintillators (TOF) will be used. Both tracks of the scattered lepton and recoiling proton will be measured in coincidence by the BLAST detector. The left-right symmetry of the detector with respect to the beam axis effectively doubles the acceptance for coincident lepton-proton events, i.e. two redundant experiments are carried out simultaneously.

The BLAST wire chambers embedded in the horizontal octants of the toroid provide three tracking points of the curved track in the region of maximum magnetic field. The chambers have been restrung with new wires after arrival at DESY in summer 2010. The tracking capability will be enhanced with an additional tracking point close to the target where only little magnetic field is present. One GEM (Gas Electron Multiplier) chamber (GC) will be built in each sector for this purpose. The additional near-target tracking point will decouple the determination of scattering angle and vertex location from the momentum reconstruction and will result in improved track resolution, more powerful background suppression and a simplified analysis scheme. The internal target has been newly built, with a storage tube of $\approx 60 \mathrm{~cm}$ length and ellipical cross section, optimized for DORIS beam parameters. Also, a new vacuum scattering chamber has been designed and built allowing access to smaller scattering angles than in the previous geometry of BLAST. Much of the BLAST trigger and data acquisition system will be replaced as well.

In order to control the systematic uncertainties to the percent level, the relative luminosity of the experiment in a cycle of four combinations of beam species and magnet polarity will be measured precisely based on small-angle elastic scattering. The lepton is detected at a forward angle of $\approx 12^{\circ}$ in coincidence with the recoil proton accepted by the BLAST detector. For the lepton arm, a GEM tracking telescope based on three tracking planes is constructed for each sector, to be embedded into the forward cone between the coils of the horizontal octant and the downstream end of the 
wire chamber (see Fig. 1). Each tracking plane is built as a $10 \times 10 \mathrm{~cm}^{2}$ triple-GEM detector with stereo-strip readout, following a design similar to that of a prototype GEM detector recently realized at MIT [20]. The GEM elements will be interleaved with additional MWPC elements to add redundancy, which can be used to cross-calibrate efficiencies or to be operated as an independent luminosity monitor. The spatial resolution of better than $100 \mu \mathrm{m}$ will allow to reconstruct the lepton vertex at the extended target with a resolution of better than $1 \mathrm{~cm}$, limited by multiple scattering. The kinematics correspond to low $Q^{2} \approx 0.17(\mathrm{GeV} / \mathrm{c})^{2}$ and large $\varepsilon \approx 0.98$ at $2.0 \mathrm{GeV}$ beam energy, where the cross section is well known and where the two-photon exchange effect is expected to be negligible. The $e^{+} p$ and $e^{-} p$ cross sections are hence expected to be equal and the ratio of count rates becomes a monitor of the respective luminosity ratio. With the given luminosity of $2 \cdot 10^{33} /\left(\mathrm{cm}^{2} \mathrm{~s}\right)$ the statistical error of this monitor will be better than $1 \%$ in five hours at $4.5 \mathrm{GeV}$, with at least a factor 20 higher counting statistics at $2.0 \mathrm{GeV}$. It is anticipated that the beam will be switched between positrons and electrons and the magnet polarity be reversed of the order once per day. By cycling through four states, the detection efficiencies for lepton and proton detection cancel out to first order. This way, about 100 random cycles through the four running states will substantially reduce systematic uncertainties associated with slowly varying control parameters such as detector efficiencies or field drifts.

Figure 2 shows the projected statistical uncertainties for the $e^{+} p$ to $e^{-} p$ cross section ratio at a beam energy of 2.0 $\mathrm{GeV}$ as a function of virtual photon polarization along with previous data [13] and various theoretical expectations [5, $6,7,8,9,10,11]$. The systematic uncertainties of the ratio are expected to be less than $1 \%$.

To summarize, the proposed OLYMPUS experiment at the lepton storage ring DORIS at DESY will provide a definitive determination of the two-photon exchange effect by precisely measuring the $e^{+} p / e^{-} p$ unpolarized cross section ratio up to $Q^{2}=2.2(\mathrm{GeV} / \mathrm{c})^{2}$ and virtual photon polarization down to $\varepsilon=0.37$.

This work has been supported by the National Science Foundation under grants PHY-0855473 and PHY-0959521.

\section{REFERENCES}

1. A.J.R. Puckett et al., Phys. Rev. Lett. 104, 242301 (2010); V. Punjabi et al., Phys. Rev. C 71, 055202 (2005); Erratum-ibid. Phys. Rev. C 71, 069902(E) (2005) superseding M. Jones et al., Phys. Rev. Lett. 84, 1398 (2000); O. Gayou et al., Phys. Rev. Lett. 88, 092301 (2002); O. Gayou et al., Phys. Rev. C 64, 038202 (2001).

2. M. Meziane et al., arXiv:1012.0339v1 [nucl-ex].

3. I.A. Qattan et al., Phys. Rev. Lett. 94, 142301 (2005); M.E. Christy et al., Phys. Rev. C 70, 015206 (2004); R.C. Walker et al., Phys. Rev. D 49, 5671 (1994); L. Andivahis et al., Phys. Rev. D 50, 5491 (1994); G.G. Simon et al., Nucl. Phys. A333, 381 (1980); F. Borkowski et al., Nucl. Phys. A222, 269 (1974); Nucl. Phys. B93, 461 (1975); J.J. Murphy, Y.M. Shin, and D.M. Skopik, Phys. Rev. C 9, 2125 (1974); W. Bartel et al., Nucl. Phys. B58, 429 (1973); C. Berger et al., Phys. Lett. B35, 87 (1971); J. Litt et al., Phys. Lett. B31, 40 (1970); T. Janssens et al., Phys. Rev. 142, 922 (1966).

4. Y.S. Tsai, Phys. Rev. 122, 1898 (1961); L.W. Mo, Y.S. Tsai, Rev. Mod. Phys. 41, 205 (1969); Y.S. Tsai, SLAC report, no. SLAC-PUB-848, 1971 (unpublished); L.C. Maximon, J.A. Tjon, Phys. Rev. C 62, 054320 (2000).

5. Y.C. Chen, C.W. Kao, and S.N. Yang, Phys. Lett. B652, 269 (2007).

6. J. Guttmann, N. Kivel, M. Meziane, and M. Vanderhaeghen, arXiv:1012.0564v1 [hep-ph].

7. P.G. Blunden, W. Melnitchouk, and J.A. Tjon, Phys. Rev. Lett. 91, 142304 (2003); Phys. Rev. C 72, 034612 (2005); S. Kondratyuk, P. Blunden, W. Melnitchouk, and T.A. Tjon, Phys. Rev. Lett. 95, 172503 (2005).

8. D. Borisyuk and A. Kobushkin, Phys. Rev. C 78, 025208 (2008); M. Gorchtein, Phys. Lett. B644, 322 (2007).

9. Y.C. Chen, A.V. Afanasev, S.J. Brodsky, C.E. Carlson and M. Vanderhaeghen, Phys. Rev. Lett. 93, 122301 (2004); A.V. Afanasev, S.J. Brodsky, C.E. Carlson, Y.C. Chen, and M. Vanderhaeghen, Phys. Rev. D 72, 013008 (2005).

10. N. Kivel and M. Vanderhaeghen, Phys. Rev. Lett. 103, 092004 (2009).

11. P.A.M. Guichon and M. Vanderhaeghen, Phys. Rev. Lett. 91, 142303 (2003); M.P. Rekalo and E. Tomasi-Gustafsson, Eur. Phys. J. A 22, 331 (2004).

12. Yu.M. Bystritskiy, E.A. Kuraev, E. Tomasi-Gustafsson, Phys. Rev. C 75, 015207 (2007).

13. D. Yount and J. Pine, Phys. Rev. 128, 1842 (1962); A. Browman, F. Liu, and C. Schaerf, Phys. Rev. 139, B1079 (1965); J. Mar et al., Phys. Rev. Lett. 21, 482 (1968); B. Bouquet et al., Phys. Lett. B26, 178 (1968).

14. A Proposal to Definitively Determine the Contribution of Multiple Photon Exchange in Elastic Lepton-Nucleon Scattering (2008); Technical Design Report for the OLYMPUS Experiment (2009); http : / / web . mit . edu/OLYMPUS.

15. D. Hasell et al., Nucl. Instr. and Methods in Physics Research A603, 247 (2009).

16. C.B. Crawford et al., Phys. Rev. Lett. 98, 052301 (2007).

17. E. Geis et al., Phys. Rev. Lett. 101, 042501 (2008).

18. J. Arrington et al., Two photon exchange of electrons/positrons on the proton, Proposal at VEPP-3, nucl-ex/0408020 (2004).

19. J. Arrington et al., Beyond the Born Approximation: A Precise Comparison of $e^{+} p$ and $e^{-} p$ Elastic Scattering in CLAS, PR04-116, PAC26 (2004).

20. F. Simon et al., Nucl. Instr. and Methods in Physics Research A598, 432 (2009). 